\title{
Microfluidic Automation Using Elastomeric Valves and Droplets: Reducing Reliance on External Controllers
}

\author{
Sung-Jin Kim, David Lai, Joong Yull Park, Ryuji Yokokawa, and Shuichi Takayama*
}

\begin{abstract}
This paper gives an overview of elastomeric valve- and droplet-based microfluidic systems designed to minimize the need of external pressure to control fluid flow. This Concept article introduces the working principle of representative components in these devices along with relevant biochemical applications. This is followed by providing a perspective on the roles of different microfluidic valves and systems through comparison of their similarities and differences with transistors (valves) and systems in microelectronics. Despite some physical limitation of drawing analogies from electronic circuits, automated microfluidic circuit design can gain insights from electronic circuits to minimize external control units, while implementing highcomplexity and high-throughput analysis.
\end{abstract}

\section{Introduction}

Two key features of microfluidics are small volumes and fluid flows. These two traits give rise to many compelling advantages for microfluidic bioassays such as: reduced reagent use, rapid mixing for reactions and assays, rapid serial analysis such as in droplet microfluidics, spatio-temporal

Dr. S.-J. Kim, D. Lai, Prof. R. Yokokawa, Prof. S. Takayama

Department of Biomedical Engineering

University of Michigan

Ann Arbor, MI, 48109, USA

E-mail: takayama@umich.edu

Prof. J. Y. Park

School of Mechanical Engineering

Chung-Ang University

Seoul, Republic of Korea

Prof. R. Yokokawa

Department of Microengineering

Kyoto University

Yoshida-honmachi, Sakyo, Kyoto, 606-8501, Japan

Prof. S. Takayama

Department of Macromolecular Science and Engineering

University of Michigan

Ann Arbor, MI, 48109, USA

Division of Nano-Bio and Chemical Engineering WCU Project

UNIST, Ulsan, Republic of Korea

DOI: $10.1002 / \mathrm{smll} .201200456$ micropatterning of biochemical for cellular analysis, and recreating physiological fluid mechanical microenvironments. ${ }^{[1-4]}$ These benefits and well-established microfabrication technology make microfluidic devices promising for high-throughput and complex analysis. Accordingly, microfluidic devices are becoming increasingly sophisticated, integrating increasingly larger numbers of passive components such as channels and chambers as well as components that need active control such as valves and regulators. While the cost associated with increasing the number of microfluidic component features in these complex devices is small, the cost associated with increasing the number of the external control units to operate the microfluidic components is still large because even single external pressure control units such as a pump is relatively expensive. With increased recognition of this control-challenge that hampers broader use of microfluidic devices for high-throughput and high-complexity analysis, microfluidic engineers are searching for new ways to enable sophisticated on-chip control without increasing the number of large external controllers.

In this Concept paper, we focus on schemes to minimize the use of off-chip mechanical pressure controllers in elastomeric valve and droplet microfluidic devices for highthroughput analysis. Other microfluidic control schemes also have the potential to minimize mechanical pressure controllers: Interested readers can find good reviews or studies on centrifugal, ${ }^{[5]}$ capillary microfluidics, ${ }^{[6]}$ and microfluidic control using viscoelastic polymer solutions. ${ }^{[7]}$ On the other hand, digital microfluidics by electrowetting ${ }^{[8]}$ and electrokinetic 


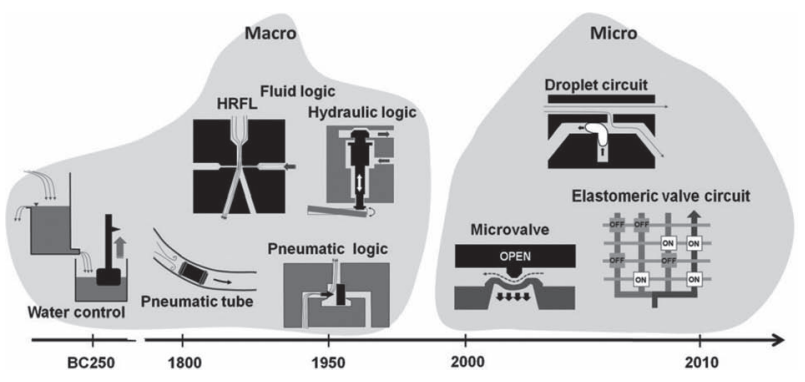

Figure 1. Timeline showing development of macroscopic and microscopic fluidic actuation and control systems.

flows ${ }^{[9]}$ are excluded because their flows are directly controlled by electricity. Also, we exclude fluidic automation schemes using pipetting robots ${ }^{[10]}$ because they extensively rely on external controllers. In Section 2, we explain the history of fluidic automation. Section 3 introduces the working principles of elastomeric valve circuits along with their biochemical applications. Section 4 presents droplet circuits focusing on key functions for autonomous rapid serial processing. To conclude, in Section 5, we discuss the outlook for design of more sophisticated automated microfluidic circuits in the near future.

\section{History of Macroscale Fluidic Control}

The history of automatic fluidic control can be traced to the legendary water clock, which was made by Ctesibius (around 250 BC), a Greek physicist and inventor. ${ }^{[11]}$ His clock was operated by a self-regulating water supply similar to the mechanism for flushing toilets nowadays. More recently, in the 1800 s to early 1900 s, pneumatic systems, or vacuum- and compressed-air-based fluidic systems, became a major mechanism for the operation and control of machinery (Figure 1). We see remnants of these control systems even today in the pneumatic tube system used to transport light and small items that must be delivered rapidly across a building such as money and medical samples. ${ }^{[12]}$

Interestingly in the $1950 \mathrm{~s}$ and $60 \mathrm{~s}$, during the transient time from pneumatic to solid-state electronic control circuitry, there was very active development of fluidic logic circuits and they can be classified into three categories: (i) high Reynolds number flow-driven fluid logic (HRFL), (ii) pneumatic logic, and (iii) hydraulic control. HRFL refers to technology that takes advantage of a jet of fluid (air or water) having high Reynolds number to create logic elements that have no moving parts. ${ }^{[13]} \mathrm{A}$ jet of fluid is to be deflected by a sidestriking control jet, resulting in non-linear amplification and other logic operations. On the other hand, pneumatic logic particularly refers to high pressure, low mass flow, on/off control systems that utilize millimeter to centimeter scale pneumatically actuated valves. Pneumatic logic is different from HRFL in that it has pressure-actuated moving solid parts. The pneumatic logic systems are highly reliable and are still used today in specialized control settings where compressed air is the only energy source available, the danger of spark

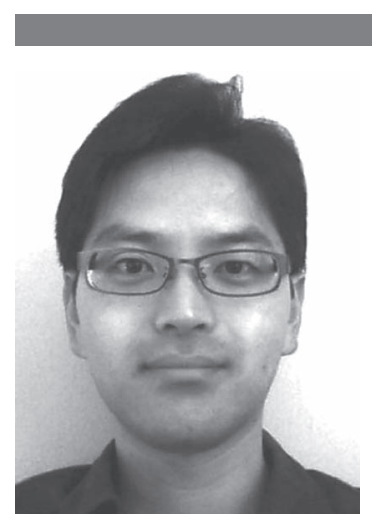

Sung-Jin Kim is a postdoctoral fellow in the Department of Biomedical Engineering at the University of Michigan since 2010 . He received his B.S. and M.S. in Mechanical Engineering from Seoul National University and KAIST in 2000 and 2002, respectively. After his 4 years' mandatory military service at Electronics Telecommunications Research Institute (ETRI), he studied Mechanical Engineering at the University of Michigan and received his Ph.D. in 2010. His research interests are microfluidic analogues to electronic circuits, capillarity control, and thermal control for micro/nano systems.

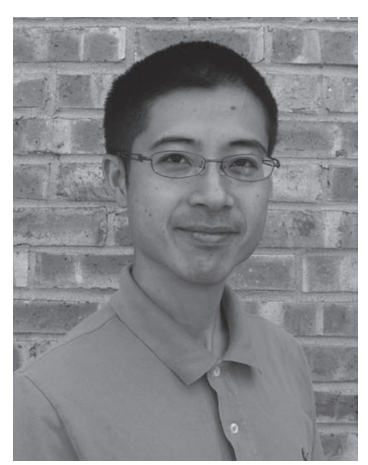

Ryuji Yokokawa is Associate Professor at the Department of Microengineering, Kyoto University, Japan, and Project Leader of Precursory Research for Embryonic Science and Technology (PRESTO), Japan Science and Technology Agency. He received his Ph.D. from the Electrical Engineering Department at The University of Tokyo in 2005, and B.S. and M.S. degrees from the Mechanical Engineering Department at Kyoto University in 2000 and 2002, respectively. He was Assistant Professor at Ritsumeikan University, Shiga (2005-2009), and Kyoto University (2009-2011). His current research areas are developing microfluidic devices for biomolecule detection and cell analysis, and nanosystems by integration of micro/nano fabrication and motor proteins.

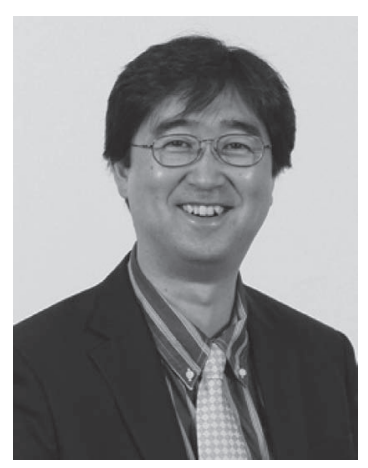

Shuichi Takayama's research interests (B.S \& M.S. from the University of Tokyo, Ph.D. from the Scripps Research Institute) started with organic synthesis. Subsequently he pursued postdoctoral studies in bioengineered microsystems at Harvard University as a Leukemia and Lymphoma Society Fellow. He is currently Professor at the University of Michigan in the Biomedical Engineering Department. He constructs microfluidic models of the body such as the oviduct, lung, and cancer metastasis. He also develops aqueous two-phase system micropatterning technologies, studies timing and rhythms of cell signaling, constructs self-switching fluidic circuits, and performs nanofluidic single-molecule DNA analysis.

generation exists, or high concentration of dust/moisture is present. ${ }^{[13]}$ Hydraulic control circuits also exist. Despite their long time delays and requirement for high operating pressures, they offer higher accuracy in delivering force and are still used today in systems such as the braking system of cars. ${ }^{[14]}$

As electronic components became more readily available, many of the fluidic controls were replaced by electronics. This transition accelerated with the development of solidstate "electronic valves"-more commonly known today as transistors-and the commercial rollout of integrated microelectronic circuits in the 1960s. Fluid logic devices normally cannot compete with their electronic counterpart because 
the size and the speed of electronic logic circuits are far more advantageous. With the increased use of microfluidics for biomolecular studies, however, the need for fluidic controls with minimal external units has also increased, leading to a renewed interest in fluidic components analogous to electronic circuits. Recently, use of a viscoelastic polymer solution, flux stabilizer, a bistable flip-flop memory, and flow rectifier have been implemented. ${ }^{[7]}$ It is important to realize that these and other microfluidic circuits developed recently are very different in size and operating mechanism from the millimeter to macroscale fluidic logic systems investigated decades ago. Much of the difference arises due to use of smaller channel sizes and in some cases also to introduction of mechanically compliant materials for device construction. Key considerations for operation of these newer generations of microfluidic devices include: (i) elasticity of the material, (ii) viscosity, and (iii) interfacial phenomena of fluids. In the next sections, we present elastomeric-valve-utilizing and droplet-based microfluidic devices that take advantage of these features. Elastomeric microfluidic devices extensively use the first two properties and droplet devices exploit the last two.

\section{Advent of Elastomeric Microfluidic Devices}

Because of the great success of microelectronics, size similarity in terms of being miniaturized, and electrohydraulic analogies, electronic circuits have inspired much of what is developed in microfluidics. With only a power source, an electronic circuit implements sophisticated autonomous functions (i.e., no aid by other external units). ${ }^{[15,16]}$ Such functions are enabled by the smart arrangement of electronic transistors, resistors, and capacitors, which are the key components of electronic circuits. Notably, an interesting analogy exits between electric and microfluidic parameters: voltage corresponds to pressure and current to flow rate. Owing to the similarity in equations between electric/electronic and microfluidic components, electric resistors correspond to microfluidic channels having fluids, capacitors to chambers having elastomeric membranes, and electronic transistors to elastomeric valves.

Such elastic property of microfluidic components comes from polydimethylsiloxane (PDMS, Young's modulus of $\sim 1 \mathrm{MPa}),{ }^{[17]}$ a widely used material for soft lithography that is to make microstructures by molding. Owing to its moderately stiff elastic property, microfluidic valves and capacitors containing PDMS membranes have appropriate operational pressure-ranges for microfluidic devices. Elastomeric valves are suitable for parallel processing of biochemicals, thereby implementing high-throughput analysis. Some of the applications like digital polymerase chain reaction $(\mathrm{PCR})^{[18]}$ need only one control source because all valves are on/off simultaneously. In many cases, however, valves should be controlled individually for sequential processes; thus typically requiring many control lines and sources. In this section, for the minimal external control, we introduce microfluidic analogues of electronic components, present their working principle, and show related biochemical applications.

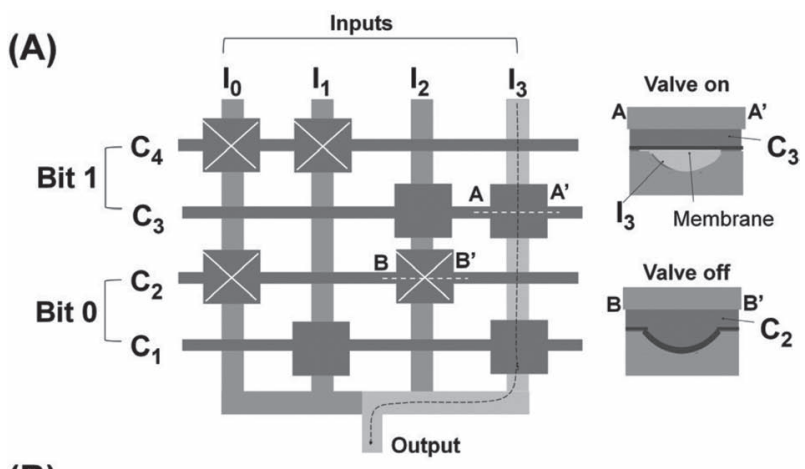

(B)

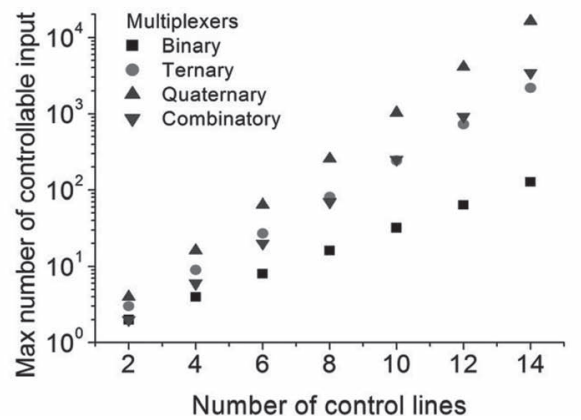

Figure 2. Microfluidic multiplexers. A) Binary multiplexer. Horizontal and vertical lines are control $\left(C_{i}\right)$ and input $\left(I_{i}\right)$ flow channels, respectively. Cross-sections ( $A A^{\prime}$ and $B B^{\prime}$ ) of the valves show that control and input liquids are in top and bottom channels, respectively, and depict on- and off-states. In each bit, on-off states of valves in the upper and lower $C_{i}$ are opposite; state 1 (or 2) is upper valves off (or on) and lower valves on (or off). The diagram shows that bit 0 and 1 are both in state 1 and thus $I_{3}$ is selected to be an output. B) Maximum number of controllable flow channels in different multiplexers.

\subsection{Key Components}

\subsubsection{Multiplexer and Demultiplexer}

A multiplexer is a component that selects one out of many possible inputs and connects that input to a single output. Control lines regulate the selection process and the number of external controllers can be significantly reduced through a smart arrangement of control lines. Figure 2A depicts the process of how one out of 4 input channels is selected by the manipulation of 4 control channels in a binary multiplexer. ${ }^{[19]}$ The vertical lines are flow input channels $\left(\mathrm{I}_{i}\right)$ transferring input liquids and the horizontal lines are control channels $\left(\mathrm{C}_{i}\right)$ containing hydraulic rectangular membrane valves. As shown in the cross-section of the multiplexer valve (Figure 2A), $\mathrm{I}_{i}$ is formed under membrane valves of $\mathrm{C}_{i}{ }^{[20]}$ Note that a particular valve (shown in squares of the right panel) is considered "on" when it is open with regards to flow through $\mathrm{I}_{i}$. Two $\mathrm{C}_{i}$ constitute one bit. Note that, in each bit, on-off states of valves in the two $\mathrm{C}_{i}$ are opposite; for example, in bit 0 , when valves in $\mathrm{C}_{1}$ are on, valves in $\mathrm{C}_{2}$ are off. Thus, each bit has only two states (state 0 and 1 ); for instance, in bit 0 , state 0 is valves-off in $\mathrm{C}_{1}$ and valves-on in $\mathrm{C}_{2}$ and state 1 is $\mathrm{C}_{2}$ off and $\mathrm{C}_{1}$ on. Figure $2 \mathrm{~A}$ shows bit 0 and 1 are both in state 1 so $\mathrm{I}_{3}$ is selected to be output (i.e., $1 \times 2^{0}+1 \times 2^{1}=3$ ). Because of this binary characteristic and a smart arrangement 
of valves, $n$ control lines (or $n / 2$ bits) can regulate up to $2^{n / 2}$ flow channels. A greater saving of the control lines can be achieved by using a ternary, a quaternary, and a combinatory multiplexer that can regulate up to $3^{n / 2}, 4^{n / 2}$, and $n ! /((n / 2) !)^{2}$ flow channels, respectively, with $n$ control lines (or $n / 2$ bits). ${ }^{[21,22]}$ The efficiency of the multiplexers is summarized in Figure 2B.

Compared to the multiplexer, a demultiplexer functions conversely. That is, it connects a single input channel to a selected output channel out of multiple possible outlets; thus working as an output selector. As a result, input and output channels in a demultiplexer are simply reversed from that of a multiplexer. ${ }^{[23]}$ Even with the use of multiplexers and demultiplexers, multiple control sources are still necessary to address many flow channels individually. Additionally, only 1 input or output can be selected at a time making this type of control unsuitable for some applications. In the next section, we show that logic gates in combination with multiplexers and demultiplexers have potential to save more control line resources.
(A)

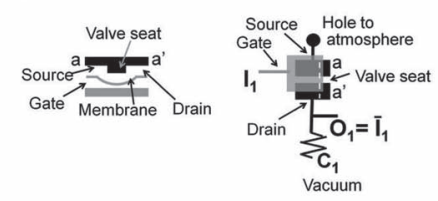

(D)

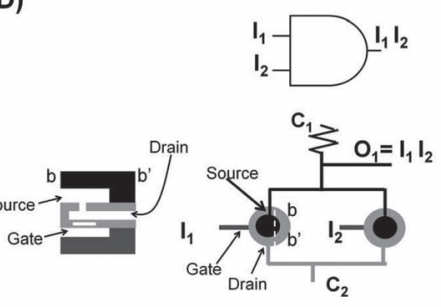

(B)

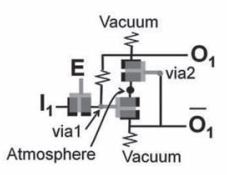

(E)

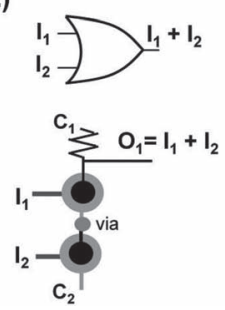

(C)

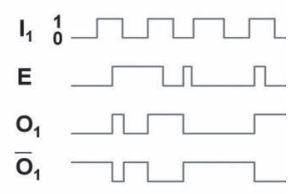

(F)
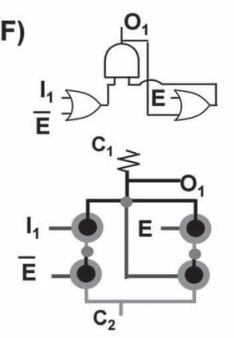

Figure 3. Microfluidic logic gates. A) Pneumatic NOT gate. Cross-section of a valve shows the valve-on state; source and drain terminals are in the top channel; and a gate terminal locates at the bottom channel under the membrane. In the top view of the valve, black area is in top channel and gray area is in bottom channel. B) Pneumatic gated D latch consisting of NOT gates. C) State of each line in gated D latch. Vacuum and atmospheric pressure are defined as state 1 and 0 , respectively. D) Hydraulic AND gate. Cross-section shows a normally open valve, which has a pressure gain with a rigid disk (white bar above gate in left panel); valve is off when gate pressure is sufficiently high to deflect the membrane containing the disk upward and to close a hole. In the top view of the valves, black, light gray, and dark gray areas are in the top, middle, and bottom channels, respectively. E) Hydraulic OR gate. F) Hydraulic static latch consisting of AND and OR gates. State of each line coincides that in (C). Note that high and low pressures are defined as state 1 and 0 , respectively, and it is the opposite of pneumatic logic.

\subsubsection{Logic Gates for Boolean Operations}

Logic gates are components that implements Boolean functions such as AND, OR, NOT, and XNOR operations. ${ }^{[24]}$ They are the elementary building blocks of digital circuits. The power of digital circuits is that more complex components like shift registers can be implemented by cascading logic gates, theoretically enabling the control of unlimited number of outputs with a set small number of control signal inputs. Both pneumatic and hydraulic logic systems have been implemented microfluidically. Both logic systems have the capability to control a biochemical analysis unit when the logic systems and the analysis unit are monolithically integrated in a device.

A key element of the microfluidic pneumatic logic (i.e., channels are filled with air or vacuum) is a normally closed (NC) valve. The NC valve is analogous to a p-channel enhanced mode MOSFET in that the valve has three terminals (gate, source, and drain) and the closed valve is on only when the source minus gate pressure is greater than some value (threshold pressure). ${ }^{[25]}$ As shown in Figure 3A, source and drain terminals are in the same plane (see left panel or black areas of right panel), but there is a valve seat between them (left panel); the gate terminal is the only access port to the bottom chamber (gray area of right panel) that is under the membrane, the gray line is connected to the gate, and the black lines are linked to source and drain. NC valves can directly be pneumatic NOT gates ${ }^{[26,27]}$ depending on their pressure connections. As shown in the right panel of Figure 3A, gate is used as an input $\left(\mathrm{I}_{1}\right)$, and drain is connected to control $\left(\mathrm{C}_{1}\right)$ at a constant vacuum and to output $\left(\mathrm{O}_{1}\right)$ channel. We define positive and vacuum pressures as state 0 and 1 , respectively. When the pressure of $I_{1}$ is positive (state 0 , i.e., pushing membrane upward), $\mathrm{NC}$ valves is off (closed), making $\mathrm{O}_{1}$ at vacuum (state 1 ). On the other hand, when $\mathrm{I}_{1}$ channel is at high vacuum (state 1 ), NC valve is on (open) because source pressure is higher than gate pressure (i.e., pushing membrane downward). At that moment, because fluidic resistance in $\mathrm{C}_{1}$ is designed to be greater than that in $\mathrm{O}_{1}$ channel, air dominantly flows to $\mathrm{O}_{1}$ channel thus making the pressure of $\mathrm{O}_{1}$ positive (state 0 ). As a result, pressure states of $\mathrm{I}_{1}$ and $\mathrm{O}_{1}\left(=\overline{1}_{1}\right)$ are always opposite; thus making the unit an analogue of the electronic NOT gate.

The more logic units are cascaded, the more sophisticated a system can be implemented. For example, two NOT gates can make a gated D latch (Figure 3B) and its behavior is illustrated in Figure $3 \mathrm{C}$. In contrast to a single NOT gate, $\mathrm{I}_{1}$ is connected to the source terminal of a valve. As long as clock pulse $(\mathrm{E})$ is in state 1 (i.e., the valve is on for $\mathrm{I}_{1}$ ), $\mathrm{O}_{1}$ follows $\mathrm{I}_{1}$ because $\mathrm{O}_{1}$ is directly connected to $\mathrm{I}_{1}$ through the valve controlled by $\mathrm{E}$. Note that even after $\mathrm{E}$ is in state $0, \mathrm{O}_{1}$ maintains (i.e., latches) the original $\mathrm{I}_{1}$ state when $\mathrm{E}$ is in state 1 . This is because, as shown in Figure $3 \mathrm{~B}, \mathrm{O}_{1}$ and $\mathrm{O}_{1}$ bar can retain their original states when the valve controlled by $\mathrm{E}$ is off. As such, cascading structures with 23 NOT gates can be made to constitute a 4-bit shift register, which can decode the sequential inputs from a single port into 4 parallel output ports. ${ }^{[26]}$ When a shift register's outputs are connected to the control lines of a multiplexer, the outputs can regulate the control lines. Theoretically, by increasing the number of bits a shift register can handle, an arbitrarily large number of control 
lines can thus be regulated using the same number of external control units.

The basic building block of the hydraulic logic gates (i.e., channels are filled with liquids) is a normally open (NO) valve; it is analogous to an electronic p-channel junction gate field-effect transistor and makes a pressure gain. ${ }^{[28]}$ Note that definition of state in hydraulic logic is the opposite of that in the pneumatic logic just described. That is, high pressure $(\sim 100 \mathrm{kPa})$ and atmospheric pressure are state 1 and 0 , respectively. Figure 3D shows an AND gate; dark gray, light gray, and black lines in the right bottom panel are bottom, middle, and top channels, respectively; and when gate pressure is high (state 1), the membrane containing a stiff disk (marked in white of left bottom panel) deflects upward and flow from the top to middle channel is prevented (valve-off). Control line $\mathrm{C}_{1}$ is always at high pressure and $\mathrm{C}_{2}$ is at atmospheric pressure. Only when the two inputs $\left(\mathrm{I}_{1}\right.$ and $\left.\mathrm{I}_{2}\right)$ are both at high pressures (state 1), thus closing the two NO valves (off), does the output also take on state 1 . The OR gate can be defined in a similar way (Figure 3E). A static latch consists of two OR and one AND gate (Figure 3F). Here the state of each input or output $\left(\mathrm{I}_{1}, \mathrm{E}\right.$, or $\left.\mathrm{O}_{1}\right)$ is similar to that of the pneumatic latch (Figure 3C). The difference is that the hydraulic static latch needs two clock inputs ( $\mathrm{E}$ and $\mathrm{E}$ bar) but have one output $\left(\mathrm{O}_{1}\right)$. The advantage of the hydraulic logic over the pneumatic logic is that it prevents potential bubble formation due to the lack of a vacuum line and latching times can be permanent. However, as the hydraulic logic system serially cascades more logic gates, it suffers significant pressure drops compared to pneumatic logic because viscosity of water is two orders of magnitude greater than that of air.

\subsubsection{Constant-Input-Driven Oscillators}

A constant-input-driven oscillator is a device that converts constant input (flow or pressure) to an oscillatory output flow. Because the device does not need dynamic external control units such as solenoid valves, it can simplify the need of off-chip control units. The generated output can be used as an on-chip timing source for microfluidic logic gates. Also, when a biochemical solution is used as an input, the output itself can be used to directly manipulate biomolecules. To date, constant flow- and pressure-driven oscillators have been presented.

A constant-flow-driven oscillator uses $\mathrm{NC}$ valves, which are analogous to p-type metal-oxide-semiconductor fieldeffect transistors (pMOSFETs), as a key element. ${ }^{[29,30]}$ Figure 4A shows how the drain and gate terminals of two $\mathrm{NC}$ valves (the $\mathrm{NC}$ valve structure is shown in Figure 3A) are connected to each other to make the two valves alternate on-off states with an opposite phase to each other. This happens because when the difference between the source and gate pressure surpasses a threshold pressure causing a valve to open and release its pressure, the flow output increases the gate pressure in the other valve causing it to close. Then, owing to the constant inflow, the pressure in the newly closed valve accumulates until the pressure surpasses a threshold value at which point the valve opens increasing the gate pressure in the other valve and closing it. In this way, the two valves open and close alternately. To control the duty cycle of the outflow, the opening width $\left(w_{\mathrm{o}}\right)$ of the two valves can be made asymmetric. This geometrical difference in the valve design changes the threshold pressure of opening for each valve. This in turn causes the pressure accumulation time of the two valves to become different, thereby changing the duty cycle or the relative duration that each valve is on. ${ }^{[30]}$

A constant-pressure-driven oscillator uses a free-standing flexible disk (Figure 4B). ${ }^{[31]}$ Because the disk edge is not bonded to the device, flow passes through the disc edge when the disc deflects. Significant inertial force of flow initiates at a high-pressure input $(70 \mathrm{kPa})$ and, by the interaction of the elastic force of the disk, vibration of the disk occurs, thereby oscillating the outflow $(\sim 100 \mathrm{~Hz}$ at the order of $1 \mathrm{~mL} / \mathrm{min}$ flow rates). Another constant-pressure-driven oscillator is a pneumatic ring resonator, which uses cascaded odd numbers of NOT gates using NC valves. ${ }^{[32]}$ The drains of the valves are connected to a common atmospheric pressure and the other gate terminal. Because of the pressure condition and the internal capacitance of each valve, the membranes (or drain pressures) of the valves oscillate alternately and the frequency range is reported at $1-100 \mathrm{~Hz}$. The ring oscillator is used as an on-chip timing source for a finite-state machine capable of sequential metering, mixing, and flushing. ${ }^{[33]}$ The operating oscillation frequency of two constant-pressure-driven oscillators are fast $(\sim 100 \mathrm{~Hz})$ compared to that of the constant-flowdriven oscillators $(<\sim 1 \mathrm{~Hz})$. It is because these pressure-driven oscillators either have very high operational pressure ranges owing to utilization of inertial effects ${ }^{[31]}$ or use air ${ }^{[32]}$ that gives rise to much lower fluidic resistance than that of water.

\subsubsection{Other Useful Components}

A device that controls the flow-rate ratio of two outflows using a single periodic pressure source has been described. ${ }^{[34]}$ In this device, two flow channels merge at the outlet and 
each flow channel has two membrane fluidic capacitors serially connected through fluidic resistors and a NC valve. In each flow channel, a combination of two capacitors work as a bi-directional pump at a specific range of pressure excitation frequency because the second capacitor functions like a bandpass filter. The frequency range can be tuned by the product of capacitance and resistance in the channel. The NC valve changes the bi-directional flow into a uni-directional flow because the gate is at atmospheric reference pressure and source pressure of the valve alternates between positive and negative values. As a result, when two channels have different ranges of working excitation pressure frequency, the flow-rate ratio of the two outflows is controlled by the excitation frequency.

Another interesting component is a peristaltic micropump, which uses a single periodic pressure source. ${ }^{[35]}$ The device has different size, quasi NC valves connected in series through source and drain terminals, and the gate terminals are linked to each other. The smaller valves have faster response times owing to the small, internal membrane capacitance. Because the size of the connected valve increases (or decreases) along the flow direction, the valves close sequentially by the arrival of a single pressure pulse, thus enabling pumping.

\subsection{Biochemical Applications}

To date, compelling biochemical applications using microfluidic logic gates, oscillators, frequency-specific flow controllers are not reported. This may be because such components are at a primitive stage and they have limited operational ranges and flexibility compared to those extensively depending on external controllers. On the other hand, with the combination of external controllers, microfluidic multiplexers using elastomeric valves have been widely used for the parallel processing of biochemicals. An initial compelling application of the multiplexers was protein crystallization. ${ }^{[36,37]}$ For crystallization, metering and mixing of reagents while keeping a constant nL-scale reaction-volume are essential. ${ }^{[36]}$ Owing to the microscale of the devices' chamber, the assay can highly concentrate precise amounts of target molecules and can mix reagents rapidly; more recently, these advantages have also been utilized in the study of protein binding kinetics. ${ }^{[38,39]}$ Compared to the crystal growth of conventional microbatch and hanging-drop formats, the device implemented faster growth and higher hit rate of crystals. The device also measured phase behavior of xylanase crystallization systematically (Figure 5A-D). ${ }^{[37]}$ Two 4-bit binary multiplexer arrays (Figure 5A) select one of 16 reagents and 16 buffers separately, thereby enabling 144 parallel chemical reactions. The selected solutions are metered and injected as consecutive slugs ( $\sim 80 \mathrm{pL}$ each) through a positive displacement cross-injection junction (Figure $5 \mathrm{~B}$ ) into the ring structure (Figure $5 \mathrm{C}$ ) and then mixed in less than $3 \mathrm{~s}$ (Figure 5D). Because such a process is done systematically, protein phase-space for crystallization (Figure 5E) was completely mapped efficiently.

Multiplexers can also be used for cell culture systems: Gomez-Sjoberg et al. developed an automated cell culture and screening system that provide arbitrary culture media in
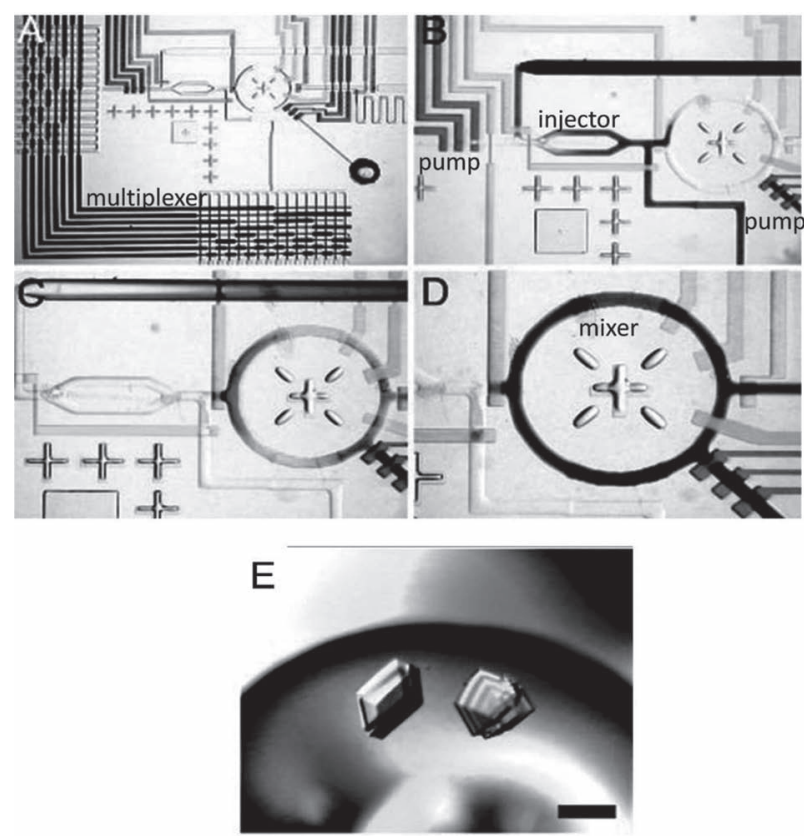

Figure 5. Applications of microfluidic multiplexers. A-D) Optical micrographs of "Formulation chip". E) Large, single, 3D crystals grown directly from optimal screen. A-E, reproduced with permission. ${ }^{[37]}$ Copyright 2004, National Academy of Sciences, USA.

96 chambers having $60 \mathrm{~nL}$ individual volume. ${ }^{[40]}$ Composition of the media provided through 16 inputs could be changed with high temporal resolution. Human primary mesenchymal stem cells (hMSCs) were cultured for weeks to examine effects of osteogenic stimulation on differentiation, proliferation, and cell motility. Multiplexers can also be applied to other cell applications such as a single-cell analysis ${ }^{[41-43]}$ and arrayed cell analysis. ${ }^{[44,45]}$ In single-cell analysis, each cell is seeded in parallel multiple chambers individually and its response is individually analyzed; thus enabling cyclopedic data collections. For detection of target intracellular materials such as DNA or mRNA, they are extracted and their concentrations are increased through PCR to a detectable level. ${ }^{[41,42]}$

\section{Droplet Circuits}

Droplet circuits are appropriate for rapid serial processing of biochemicals because the droplet generation frequency can be over a $\mathrm{kHz}$ and subsequent droplet processing and analysis can also be rapidly performed. Many factors affect generation of droplets. ${ }^{[4]}$ Important factors are size and shape of channel junctions, viscosity of immiscible phases, interfacial tension between phases and channel walls. For example, between two phases, a less viscous phase tends to be droplets such as air droplets in water and water droplets in oil. Typically, high interfacial tension between two phases is necessary for spontaneous droplet formation; to make droplets in two phases that have low interfacial tension like aqueous twophase system, additional mechanical perturbations are necessary. ${ }^{[47,48]}$ Also, like water (or oil) droplets in a hydrophobic 
(or hydrophilic) channel, a relatively less wettable channel with respect to the phase that forms the droplets is necessary. After the generation of droplets, other serially connected components perform key functions. In this section, we introduce key functions of droplet circuits. Although some of the functions are not directly analogous to that of electronic circuits, the overall system is inspired by electronic circuits in that the modularity of each component is essential for the serial processing. We briefly discuss their working principle and show how they are used in the overall system for rapid biochemical processing.

\subsection{Key Components}

\subsubsection{Droplet Sorter, Mixer, and Splitter}

Passive sorting mechanisms are based on droplet size and weight. Sorting of different size droplets can use channel geometry that vary the ratio of shear forces. ${ }^{[49]}$ When a droplet meets bifurcating channels, it flows into a branch with a relatively high shear force because the force drives the droplet into the branch instantaneously. Small droplets select low-resistance channel because higher flow rate of the channel results in high shear force (left panels of Figure 6A). On the other hand, with increasing droplet size, the droplet gets higher shear force toward the high-resistance channel because droplet's effective area to that direction increases. Accordingly, large droplet flows into the high-resistance channel (right panels of Figure 6A). A device using gravity also works for sorting of the droplets having different size and weight. ${ }^{[50]}$ Despite its simplicity and high efficiency, the device requires relatively small sample velocities to allow gravity to take effect over designed flow distances (sedimentation of $200 \mu \mathrm{m}$ over $2.5 \mathrm{~cm}$ in channel length at $1 \mathrm{~mL} / \mathrm{h}$ sample velocity). Droplet sorting is also achieved via electric fields that generate a force to steer droplets into branching downstream channels depending on its fluorescence output, ${ }^{[51]}$ and complex traffic operations were achieved with droplet sorting into five individual channels. ${ }^{[52]}$ In addition, passive droplet rearrangement can be achieved with a channel loop design. ${ }^{[53]}$ For example, a droplet traveling in series ABCDE can be rearranged to be ACDBE through the change of flow resistance in channels.

At high interfacial tension, droplet fusion will occur spontaneously with channel geometry ${ }^{[54,55]}$ between the two droplets containing different reactants (Figure 6B). Further rapid mixing $(\sim 2 \mathrm{~ms})$ is achieved through chaotic advection through a long serpentine channel (Figure 6C). ${ }^{[56]}$ Using bifurcating channels or $\mathrm{T}$ channels, a passive splitter makes a monodisperse mother droplet into evenly split two sister droplets. ${ }^{[57]}$ The ratio of droplet volume splitting is roughly equal to daughter channel length ratio or inversely proportional to the daughter channel flow rate. This process can be repeated numerously in series achieve the desired droplet number or droplet size for both single and double emulsions (Figure 6D). ${ }^{[58]}$ Droplets can also be actively split or mixed repeatedly via electrofusion, ${ }^{[59]}$ one that is advantageous for mixing droplets at low interfacial tensions. Although active forms of droplet operations are capable of complex droplet manipulations, ${ }^{[60]}$ external control technologies have yet to produce droplet operations at the rate of flow-focusing droplet generation.

Another recent passive operation is the passive droplet dilutor. This technique is capable of high-throughput dilution and screening of nanoliter droplets via a passively activated side valve geometric design ${ }^{[61]}$ (pillar structures of Figure 6E). A large droplet is trapped in a dilution chamber and forms the mother droplet containing contents to be diluted. Subsequent input droplets contain the diluent that fuses with the mother droplet, thus diluting the mother droplet. The increased volume of the mother droplet closes the side valve, and shear force to the downward channel increases thereby generating output droplet from the mother droplet until the side valve is re-opened. As this process repeats, concentrations of mother and output droplets are significantly diluted with high precision. 


\section{1.3. Traffic Control via Boolean Operations, Flip-Flop Switching, and Synchronization}

Other droplet microfluidic analogues of electronic circuits such as logic gates, switches, and synchronizers make elegant use of channel geometry (Figure 6F). ${ }^{[62]}$ The existence of a droplet affect the flow of the other droplet, thereby enabling Boolean functions. The working principle is similar to the passive sorter in that designed flow resistance and shear force govern the traffic of the droplets, thus implementing embedded functions. Further characterization of the synchronizer geometry was studied by Ahn et al. ${ }^{[63]}$ and is useful for synchronizing droplets for downstream droplet fusion. In the synchronizer, the leading droplet experiences higher resistance than the lagging droplet, allowing for the lagging droplet to have higher velocity and catch up. When the droplets are synchronized, the pressures differences stabilize and the droplets exit the ladder network synchronized and traveling at the same velocity.

\subsection{Biochemical Applications}

Droplet microfluidics is well suited for the applications that require rapid analysis such as high-throughput singlecell analysis, protein analysis, and PCR requiring ultrafast reaction times. Rapid single-cell analysis can be performed through the sequence of single-cell encapsulation, exposure to stimulant of choice via droplet fusion, short or long-term incubation times and finally assay and signal acquisition (Figure 7). ${ }^{[64]}$ The advantages of such applications are the ability to keep precise amounts of reactants separate until the desired conditions are met for them to be mixed and after mixing as started, the small diffusion lengths allow for rapid reaction times for high-throughput functionality. Through the use of a few simple electrodes, complex operations can be performed on protein for a multi-step assay with fluorescent readout. ${ }^{[64,65]}$ This device greatly simplifies an otherwise laborious laboratory protocol at a greatly reduced cost on both operator cost and material costs through automation and miniaturization. Similarly through automation and miniaturization, droplet microfluidics is capable of performing a commonly used technique: PCR with 1.5 million amplifications in parallel at over $99 \%$ accuracy. ${ }^{[66]}$ Purified genomic DNA and reactants necessary for PCR are encapsulated into droplets at the specified concentrations. Using mostly passive operations and a simple electrode for electrofusion, the device enables complex droplet operations and droplet fusion to achieve appropriate delivery and mixture of droplet components at the correct sequence and conditions for PCR to occur.

Owing to its relatively simple device geometry, droplet microfluidic devices are relatively easy to design, serially cascade, and fabricate. However, the device may be inappropriate for applications that need to keep target biomolecules in the droplet while removing and changing solutions, and for assays requiring immobilization of biomolecules on channel surfaces. Although elastomeric microfluidic devices are conceptually more complex and need more expertise to

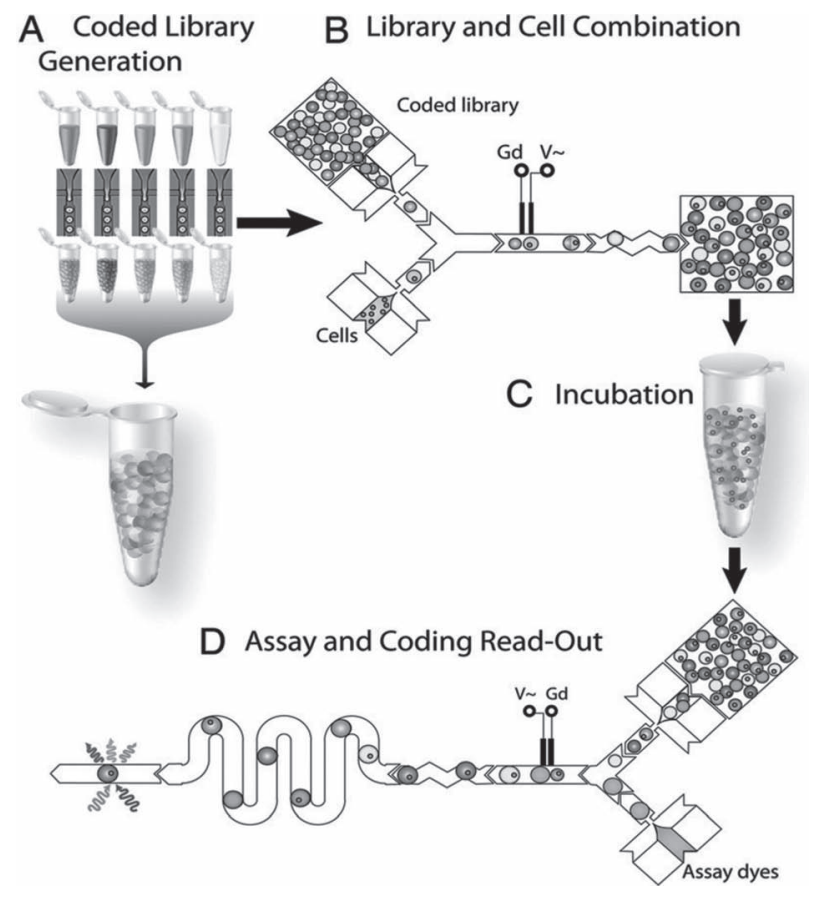

Figure 7. Droplet microfluidic application flowchart. A) Cell stimulants are encapsulated into droplets and stored off chip. B) Stimulants are mixed together or with single-cell encapsulated droplets via fusion operations. C) After fusion, the droplets can then be recollected and incubated off chip if necessary. D) Droplets are once again loaded into a droplet device with fusion operations with separate reagents for protein assay. Fluorescence signals can then be acquired downstream after chaotic advection mixing to determine effects of stimulants on single cells. Reproduced. ${ }^{[64]}$

design, they would be more suitable for these latter types of applications.

\section{Outlook and Perspectives}

Although microfluidic control circuits that are analogues of electronic circuits are in their infancy, they have the potential to minimize external control units and to perform autonomous, embedded functions. Nevertheless, to make development of such electronics-inspired microfluidic circuits more useful, we should understand the (i) operational, and (ii) physical limitations behind the analogy. There are many types of electronic circuits but some electronic circuit designs may be more appropriate for conversion into fluidic circuits than others. Given the nice analogy between electronic and microfluidic circuits in terms of designing and analyzing microfluidic circuits, what might the analogy provide in terms of fruitful directions for future development?

In terms of operation, both elastomeric and droplet microfluidic circuits have an inherent limitation because, once such devices are fabricated, their functions and operational ranges are more restricted than those that extensively use external controllers. For example, in the microfluidic oscillator circuit, the input flow rate or pressure determines the oscillation period for a given device. In other words, flow rate and 
oscillation period cannot be independently regulated; thus limiting flexibility. Also, the range of oscillation periods is narrower compared to those relying on external controllers. This also applies to droplet circuits where flow rate in conjunction with the bifurcating channel's shape and size determine droplet size and its generation frequency. Nevertheless, when specific operational ranges are determined and the devices are designed accordingly, they will be useful like applicationspecific integrated circuit (ASIC) chips in electronics.

Physical limitation exists in elastomeric microfluidic circuits. To perform even relatively simple digital functions using Boolean operations, elastomeric valve circuits need relatively large number of valves. In the 4-bit shift resistor, $\sim 60$ valves were necessary to convert sequential signals of a single source to 4-bit parallel signals. As the number of Boolean operations increase, as required for more sophisticated functions, the number of serially connected valves drastically increases due to the nature of the modular approach (functional partition by elementary components). In electronic circuits, however, this serial/modular approach is plausible. For example, the Intel Itanium 2 processor, which is a microprocessor introduced in 2004, has $\sim 6 \times 10^{8}$ transistors with each having sizes on the order of $\sim 100 \mathrm{~nm} .^{[67]}$ Because electrical resistance of integrated wires and transistors can be easily regulated, the processor performs sophisticated digital computing (Boolean operation) with an acceptable internal voltage drop. In contrast, if microfluidic valves were to be fabricated with such high density and small size, control of pressure would become a significant issue. Threshold pressures of microfluidic valves would be high due to their small, stiff membranes. More importantly, the pressure drop would be enormous due to the reduced channel size and the serial connection of channels. For instance, a 10 -fold reduction of channel radius (e.g., 100 to $10 \mu \mathrm{m}$ ) results in a $10^{4}$ fold increase of flow resistance. Also, total resistance linearly increases with the increasing number of serially connected channels and valves. Such analysis suggests limits in effectively transferring digital circuit design concepts that use Boolean operations to microfluidics.

In this regard, microfluidic circuits may gain more fruitful insights from analog electronics, where electric components and transistors give continuous output voltages and currents. Microfluidic analogues of analog electronic-circuits are oscillators, ${ }^{[29-32]}$ peristaltic pumps, ${ }^{[35]}$ and frequency-specific flow controller. ${ }^{[34]}$ Notably, they have simpler structures; thus implying easier fabrication and less pressure drop. Also, their own feedback control and continuous output enable their input flows directly to be output flows. In contrast, digital microfluidic circuits using Boolean operations are designed to indirectly control biochemical solutions. ${ }^{[26-28]}$ Parallel biochemical processing with such analog microfluidic circuits, however, has not been demonstrated yet except for a very simple immunoassay. ${ }^{[29]}$ A clever arrangement of microfluidic valves, capacitors, and resistors in conjunction with selection of control input would be needed to enable more sophisticated parallel biochemical processing. Such devices can be inspired by analog electronics because they perform parallel processing with electric resistors, capacitors, and transistors.
In droplet circuits, on the other hand, a serial/modular approach is plausible and, through the approach, droplet circuits have already demonstrated several rapid/serial biochemical processing. Because each component of droplet circuits is simple and does not suffer high pressure drop, components like droplet generators, splitters, and sorters are serially connected without high pressure drops. Nevertheless, despite seemingly facile serial modularity, integrating each component can result in unexpected issues due to the relatively small actuation forces generated and susceptibility to fluctuations and disturbances. By overcoming such obstacles and finding good biochemical applications, droplet circuits have become excellent tools for rapid serial processing of biochemicals.

\section{Acknowledgements}

We thank the NIH (GM09604-01), Institutional Program for Young Researcher Overseas Visits, Japan Society for the Promotion of Science (USPS), and the Basic Science Research Program (2012R1A1A1015181), and the WCU (World Class University) program (No. R322008000200540) through the National Research Foundation of Korea (NRF) as funded by the Ministry of Education, Science and Technology (MEST) for support.

[1] P. S. Dittrich, A. Manz, Nat. Rev. Drug Discovery 2006, 5, 210-218.

[2] S. Vyawahare, A. D. Griffiths, C. Merten, Chem. Biol. 2010, 17, 1052-1065.

[3] G. M. Whitesides, Nature 2006, 442, 368-373.

[4] P. Liu, R. A. Mathies, Trends Biotechnol. 2009, 27, 572-581.

[5] a) D. Mark, S. Haeberle, G. Roth, F. von Stetten, R. Zengerle, Chem. Soc. Rev. 2010, 39, 1153-1182; b) R. Gorkin, J. Park, J. Siegrist, M. Amasia, B. S. Lee, J.-M. Park, J. Kim, H. Kim, M. Madou, Y.-K. Cho, Lab Chip 2010, 10, 1758-1773.

[6] a) A. W. Martinez, S. T. Phillips, G. M. Whitesides, E. Carrilho, Anal. Chem. 2010, 82, 3-10; b) M. R. McNeely, M. K. Spute, N. A. Tusneem, A. R. Oliphant, Proc. SPIE 1999, 3877, 210220; c) S.-J. Kim, Y. T. Lim, H. Yang, Y. B. Shin, K. Kim, D.-S. Lee, S. H. Park, Y. T. Kim, Anal. Chem. 2005, 77, 6494-6499; d) M. Zimmermann, H. Schmid, P. Hunziker, E. Delamarche, Lab Chip 2007, 7, 119-125.

[7] a) A. Groisman, M. Enzelberger, S. R. Quake, Science 2003, 300, 955-958; b) A. Groisman, S. R. Quake, Phys. Rev. Lett. 2004, 094501.

[8] a) R. Schoch, J. Han, P. Renaud, Rev. Mod. Phys. 2008, 80, 839883; b) R. B. Fair, Microfluid. Nanofluid. 2007, 3, 245-281.

[9] K. Horiuchi, P. Dutta, Lab Chip 2006, 6, 714-723.

[10] H. Erfle, B. Neumann, U. Liebel, P. Rogers, M. Held, T. Walter, J. Ellenberg, R. Pepperkok, Nat. Protoc. 2007, 2, 392-399.

[11] J. G. Landels, in Engineering in the ancient world, University of California Press, Berkeley, California 2000.

[12] G. A. Audsley, in The art of organ-building: a comprehensive historical, theoretical, and practical treatise on the tonal appointment and mechanical construction of concert-room, church, and chamber organs, Dover Publications, 1965.

[13] H. L. Stewart, in Hydraulic and Pneumatic Power for Production, Industrial Press, New York 1977. 
[14] T. Gilles, in Automotive service: inspection, maintenance repair, Delmar Cengage Learning, 2011.

[15] C. Alexander, M. Sadiku, Fundamentals of electric circuits, McGraw-Hill, New York, 2008.

[16] R. C. Jaeger, T. N. Blalock, Microelectronic Circuit Design, McGrawHill, New York, 2003.

[17] G. M. Whitesides, E. Ostuni, S. Takayama, X. Jiang, D. E. Ingber, Annu. Rev. Biomed. Eng. 2001, 3, 335-373.

[18] E. A. Ottesen, J. W. Hong, S. R. Quake, J. R. Leadbetter, Science 2006, 314, 1464-1467.

[19] T. Thorsen, S. J. Maerkl, S. R. Quake, Science 2002, 298, 580-584.

[20] D. Maier-schneider, J. Maibach, E. Obermeier, O. F. Pan, E. T. Al, J. Microelectromech. Syst. 1995, 4, 238-241.

[21] D. W. Lee, Y.-H. Cho, Lab Chip 2009, 9, 1681-1686.

[22] Z. Hua, Y. Xia, O. Srivannavit, J.-M. Rouillard, X. Zhou, X. Gao, E. Gulari, J. Micromech. Microeng. 2006, 16, 1433-1443.

[23] W. H. Grover, R. H. C. Ivester, E. C. Jensen, R. A. Mathies, Lab Chip 2006, 6, 623-631.

[24] S. G. Shiva, in Introduction to logic design, $2^{\text {nd }}$ ed, M. Dekker, New York 1998, Ch. 2 and Ch 4.

[25] H. Takao, M. Ishida, K. Sawada, J. Microelectromech. Syst. 2002, 11, 421-426.

[26] M. Rhee, M. A. Burns, Lab Chip 2009, 9, 3131-3143.

[27] E. C. Jensen, W. H. Grover, R. a Mathies, J. Microelectromech. Syst. 2007, 16, 1378-1385.

[28] J. A. Weaver, J. Melin, D. Stark, S. R. Quake, M. A. Horowitz, Nature Phys. 2010, 6, 218-223.

[29] B. Mosadegh, C.-H. Kuo, Y.-C. Tung, Y.-suke Torisawa, T. Bersano-Begey, H. Tavana, S. Takayama, Nature Phys. 2010, 6, 433-437.

[30] S.-J. Kim, R. Yokokawa, S. C. Lesher-Perez, S. Takayama, Anal. Chem. 2012, 84, 1152-1156.

[31] H. M. Xia, Z. P. Wang, W. Fan, A. Wijaya, W. Wang, Z. F. Wang, Lab Chip 2012, 12, 60-64.

[32] P. N. Duncan, T. V. Nguyen, E. E. Hui, in Proceedings of the $\mu$ TAS 2010 Conference, Groningen, Netherlands, Oct 3-7, 2010, pp. 1838-1840.

[33] T. V. Nguyen, S. Ahrar, P. N. Duncan, E. E. Hui, in Proceedings of the HTAS 2011 Conference, Seattle, USA, Oct 2-6, 2011, 741-743.

[34] D. C. Leslie, C. J. Easley, E. Seker, J. M. Karlinsey, M. Utz, M. R. Begley, J. P. Landers, Nature Phys. 2009, 5, 231-235.

[35] H. Lai, A. Folch, Lab Chip 2011, 11, 336-42.

[36] C. L. Hansen, E. Skordalakes, J. M. Berger, S. R. Quake, Proc. Natl. Acad. Sci. USA 2002, 99, 16531-16536.

[37] C. L. Hansen, M. O. A. Sommer, S. R. Quake, Proc. Natl. Acad. Sci. USA 2004, 101, 14431-14436.

[38] S. R. Bates, S. R. Quake, Appl. Phys. Lett. 2009, 95, 073705-3.

[39] S. J. Maerkl, S. R. Quake, Science 2007, 315, 233-237.

[40] R. Gómez-Sjöberg, A. A. Leyrat, D. M. Pirone, C. S. Chen, S. R. Quake, Analytical Chemistry 2007, 79, 8557-8563.

[41] R. J. Taylor, D. Falconnet, A. Niemistö, S. A. Ramsey, S. Prinz, I. Shmulevich, T. Galitski, C. L. Hansen, Proc. Natl. Acad. Sci. USA 2009, 106, 3758-3763.

[42] J. F. Zhong, Y. Chen, J. S. Marcus, A. Scherer, S. R. Quake, C. R. Taylor, L. P. Weiner, Lab Chip 2008, 8, 68-74.
[43] S. Tay, J. J. Hughey, T. K. Lee, T. Lipniacki, S. R. Quake, M. W. Covert, Nature 2010, 466, 267-271.

[44] L. M. Fidalgo, S. J. Maerkl, Lab on a Chip 2011, 11, 1612-1619.

[45] G. A. Cooksey, C. G. Sip, A. Folch, Lab on a Chip 2009, 9, 417-426.

[46] S.-Y. Teh, R. Lin, L.-H. Hung, A. P. Lee, Lab Chip 2008, 8, 198-220.

[47] I. Ziemecka, V. van Steijn, G. J. M. Koper, M. Rosso, A. M. Brizard, J. H. van Esch, M. T. Kreutzer, Lab Chip 2011, 11, 620-624.

[48] D. Lai, J. P. Frampton, H. Sriram, S. Takayama, Lab Chip 2011, 3551-3554.

[49] Y. C. Tan, Y. L. Ho, A. P. Lee, Microfluid. Nanofluid. 2008, 4, 343-348.

[50] D. Huh, J. H. Bahng, Y. Ling, H. H. Wei, O. D. Kripfgans, J. B. Fowlkes, J. B. Grotberg, S. Takayama, Anal. Chem. 2007, 79, 1369-1376.

[51] J. C. Baret, O. J. Miller, V. Taly, M. Ryckelynck, A. El-Harrak, L. Frenz, C. Rick, M. L. Samuels, J. B. Hutchison, J. J. Agresti, D. R. Link, D. A. Weitz, A. D. Griffiths, Lab Chip. 2009, 9, 1850-1858.

[52] L. Wang, L. A. Flanagan, N. L. Jeon, E. Monuki, A. P. Lee, Lab Chip. 2007, 7, 1114-1120.

[53] Y. C. Tan, J. S. Fisher, A. I. Lee, V. Cristini, A. P. Lee, Lab Chip. 2004, 4, 292-298.

[54] L. Mazutis, J. C. Baret, A. D. Griffiths, Lab Chip. 2009, 9 , 2665-2672.

[55] L. H. Hung, K. M. Choi, W. Y. Tseng, Y. C. Tan, K. J. Shea, A. P. Lee, Lab Chip. 2006, 6, 174-178.

[56] H. Song, J. D. Tice, R. F. Ismagilov, Angew. Chem. Int. Ed. 2003, $42,768-772$

[57] D. R. Link, S. L. Anna, D. A. Weitz, H. A. Stone, Phys. Rev. Lett. 2004, 92, 054503.

[58] A. R. Abate, D. A. Weitz, Lab Chip 2011, 11, 1911-1915.

[59] S. K. Cho, H. Moon, C. J. Kim, J. Microelectromech. S. 2003, 12, 70-80.

[60] P. Paik, V. K. Pamula, R. B. Fair, Lab Chip. 2003, 3, 253-259.

[61] X. Niu, F. Gielen, J. B. Edel, A. J. deMello, Nature Chem. 2011, 3, 437-442.

[62] M. Prakash, N. Gershenfeld, Science 2007, 315, 832-835.

[63] B. Ahn, K. Lee, H. Lee, R. Panchapakesan, K. W. Oh, Lab Chip. 2011, 11, 3956-3962.

[64] E. Brouzes, M. Medkova, N. Savenelli, D. Marran, M. Twardowski, J. B. Hutchison, J. M. Rothberg, D. R. Link, N. Perrimon, M. L. Samuels, P. Natl. Acad. Sci. USA. 2009, 106, 14195-14200.

[65] L. Mazutis, J. C. Baret, P. Treacy, Y. Skhiri, A. F. Araghi, M. Ryckelynck, V. Taly, A. D. Griffiths, Lab Chip. 2009, 9, 2902-2908.

[66] R. Tewhey, J. B. Warner, M. Nakano, B. Libby, M. Medkova, P. H. David, S. K. Kosopoulos, M. L. Samuels, J. B. Hutchison, J. W. Larson, E. J. Topol, M. P. Weiner, O. Harismendy, J. Olson, D. R. Link, K. A. Frazer, Nat. Biotechnol. 2009, 27, 1025-1031.

[67] www.ni.com/white-paper/8564/en, accessed on May 2012

Received: February 29, 2012 Revised: May 2, 2012 Published online: July 3, 2012 